\title{
Reducing prescribing error: competence, control, and culture
}

\author{
N Barber, M Rawlins, B Dean Franklin
}

Qual Saf Health Care 2003;12(Suppl 1):i29-i32

Medication errors are probably the most prevalent form of medical error, and prescribing errors are the most important source of medication errors. In this article we suggest interventions are needed at three levels to improve prescribing: (1) improve the training, and test the competence, of prescribers; (2) control the environment in which prescribers perform in order to standardise it, have greater controls on riskier drugs, and use technology to provide decision support; and (3) change organisational cultures, which do not support the belief that prescribing is a complex, technical, act, and that it is important to get it right. Solutions involve overt acknowledgement of this by senior clinicians and managers, and an open process of sharing and reviewing prescribing decisions.

See end of article for authors' affiliations

Correspondence to: Professor $N$ Barber, The School of Pharmacy, 2939 Brunswick Square London WCIN IAX, UK; nick@nickbarber.org
M edication error is probably the most prevalent type of medical error in both primary and secondary care. In the United States it kills 7000 patients a year ${ }^{1}$ and accounts for nearly 1 in 20 hospital admissions (a similar admission rate to that of cancer $^{2}$ ). In the United Kingdom the incidence is probably similar to that of the United States.

of all types of medication error, prescribing error is the most serious. Once an error has been made, unless detected, it will be systematically applied and can result in significant harm or death. In United Kingdom hospitals, prescribers make errors in $1.5 \%$ of prescriptions; ${ }^{3}$ and in primary care errors occur in up to $11 \%$ of prescriptions. ${ }^{4}$ Communication of prescribing information between the two sectors is also less than ideal: in one study, around half of the patients were failing to take the right medicine, correctly, a month after discharge. ${ }^{5}$ Non-adherence, in part a consequence of poor prescribing, affects $30-50 \%$ of patients taking medications for chronic conditions. ${ }^{67}$

The importance of prescribing errors is magnified by the sheer frequency of prescribing. It is the most common form of treatment in the United Kingdom National Health Service (NHS), and in the community alone 637000000 prescriptions were written in the United Kingdom in 2000 and accounted for $12.3 \%$ of NHS costs. ${ }^{8}$

Despite this prescribing is a relatively neglected skill. There is no simple, or single, solution to its improvement; rather, a range of different measures is needed in order to make a significant breakthrough.
The most frequently used explanatory model of the causes of medical error has been taken from Reason's studies of the causes of disasters in high risk industries. ${ }^{9}$ It can be described, with some simplification, as studying the disaster from three perspectives: the individual, their immediate surroundings, and the organisational culture. We have grouped our suggestions as to the sources of, and solutions to, prescribing error in three broadly comparable categories: the competence of the individual, the controls immediately around which an individual practices, and the culture of medicine as a whole.

\section{INDIVIDUAL COMPETENCE}

In the United Kingdom drugs are prescribed predominantly by doctors, although nurses and pharmacists are increasingly expected to assume prescribing responsibilities.

The poverty of teaching medical students about therapeutics in general, and prescribing in particular, is currently a major source of concern to both educators ${ }^{1011}$ and medical students themselves. ${ }^{12} 13$ The deficiencies have also been highlighted in two major national surveys. ${ }^{1415}$

Once doctors start their pre-registration training they usually learn prescribing by the accretion of shards of knowledge and then building up their own collage of skills and understanding. When interviewing doctors who had made serious prescribing errors (most of which were inappropriate choices of dose), some said that no one had taught them about doses. ${ }^{16}$ They may be told which drug to prescribe by someone senior on their team, but then would look up the dose in the British National Formulary (a pocket guide on drugs provided nationally every six months ${ }^{17}$ ), or would use the hospital's formulary. They depended on the pharmacists (and sometimes nurses) to tell them if the dose was wrong.

Doctors should be competent to prescribe before they start doing so, and their competence should be demonstrable. Surely, anything else would be a nonsense in the public's view. Before young doctors start their pre-registration house officer training (soon to be their foundation two years) they should be taught about, and tested on, the selection, use, and doses of common drugs as well as the factors that affect doses (including renal failure, which, extraordinarily, seems to be a common oversight) and how to adjust for them. Students should demonstrate their competence by being given the drug charts and medical records for a series of patients and checking the prescribing for appropriateness (a technique commonly used in the education of 
pharmacists). Doctors should then be asked to prescribe new drugs for these patients. This test would be straightforward to administer and mark; and the teaching, examination, and certification need take no more than a week.

The education of nurses about prescribing is an area of contrasts. The introduction of a new curriculum in the late 1980s removed much of nurses' education about pharmacology and therapeutics. Although the cause of this was partly a shift in educational philosophy, it also sent an implicit message about the relative importance of medicines, which was reinforced on the wards. Twenty years ago two registered nurses administered medicines to patients. This was then changed to administration by a single registered nurse. Now, nurses of any grade give medicines to their patients. And nurses (particularly in the community) now have the right to prescribe a range of drugs, once they have completed further education. Furthermore, the growth of clinical nurse specialists has lead to some becoming very competent in prescribing within their particular clinical areas, such as diabetes and critical care.

Pharmacists have now extended their training to five years. Yet they have been legally unable to prescribe although they often intervene to prevent prescribing disasters and, increasingly, write discharge prescriptions for (less adequately trained) pre-registration house officers to sign!

Hospital pharmacists detect errors in around $1.5 \%$ of prescription items written. ${ }^{3}$ Because a 550 bed hospital can generate around 10000 prescription items a week, 150 prescribing errors will occur over the period. Regression modelling has shown that the experience of the pharmacists, and the time they spend on the ward, are two significant predictors of an increased detection rate (the type of ward being the only other predictor). ${ }^{18}$ In order to detect and correct prescribing errors pharmacy departments need to be resourced so they have sufficient skilled staff, with adequate time to spend on clinical monitoring.

The way in which pharmacists feed back on the errors they detect could also be improved. At present, when prescribing errors are detected, the information is indicated, verbally, to the prescriber (usually a pre-registration house officer). This means the clinical team remains unaware of its performance as a whole, or of the competence of its staff. In future the information on prescribing errors should to be fed to the team in a structured manner and discussed openly. Pharmacists cannot continue to pass on important information, verbally, without any record of their advice. Pharmacists must record their interventions in the patient's notes. And all this should be done in a blame free manner.

Although legal barriers to prescribing by pharmacists have, in theory, now been removed, their training and responsibilities have yet to be agreed. If they are to take over more of a prescribing role in the future they will have to work in different ways, including delivering a service in hospitals at times outside the hours of 9 am to $6 \mathrm{pm}$ on weekdays. In the community their clinical input is limited. This is largely due to their isolation from the main environment in which primary care is delivered, including patients' medical records and prescribers' decision making processes. In the future, pharmacists should be fully integrated into general practice and be able to provide prescribing and adherence support services from the practice premises. Pharmacists aspiring to provide these services should have their competency tested formally.

\section{CONTROL}

We use the term "control" in its widest sense, including the control of prescribers' actions by the design and use of technology (drugs being, of course, one of society's most advanced technologies). The technologies we will consider include the prescription chart, information transfer between primary and secondary care, and the use of computerised prescribing and clinical decision support.

One of the simplest steps would be the standardisation of NHS inpatient prescription charts. At present, each NHS hospital trust designs and uses its own individual (and idiosyncratic) prescribing system. As a consequence educators of trainee doctors, nurses, and pharmacists find it almost impossible to teach practical prescribing as a generic skill applicable throughout the NHS. Standardisation would reduce the errors caused by prescribers moving between sites and erroneously using a new chart because they are unfamiliar with it. It would also allow teaching to be more effective.

Standardisation should also extend to computerised prescribing screens. In the United Kingdom computerised prescribing is near ubiquitous in primary care; and although, at present, it is available throughout less than half a dozen hospitals, its universal adoption is inevitable and many sites are experimenting with it. The last time one of the authors visited our general practitioner the locum doctor did not understand how to use the prescribing system at the practice, and so wrote a prescription by hand. What will happen if a locum cannot use the computerised prescription in accident and emergency, or if a new doctor on an intensive care unit cannot prescribe dopamine on his/her first day? There is an unassailable argument that standardisation of screens and inputs would reduce errors as doctors move between hospitals.

The commonest prescribing error is to choose an incorrect dose, yet what do we do to help young prescribers to choose the right dose? We need intelligent systems that structure information around the prescribers' needs and provide real time, intelligent decision support. Such systems also need to be updated in real time so that changes to a medicine's summary of product characteristics (that is, the properties of the drug according to its license) are immediately incorporated. Integration with patients' full medical and drug histories and the results of laboratory tests will be essential. And there is even more potential in the future. Currently, many adverse drug reactions (particularly the idiosyncratic type $\mathrm{B}$ reactions) are not predictable or preventable; and their occurrence is considered to be bad luck. This will change. Once patients' pharmacogenetic profiles are known we may be able to further individualise drug treatment by selecting more appropriate therapy and almost guarantee efficacy and safety.

Computerised prescribing systems already have great potential. In one United States hospital they reduced serious medication errors by $86 \%,{ }^{19}$ yet that potential should not be taken for granted. In a review of medication errors suffered by elective surgical patients the most error prone part of the system was the computerised prescribing of discharge medication. ${ }^{20}$ In the United Kingdom, where primary care has probably the most extensive computerised prescribing in the world, a recent study of the prevalence of preventable drug related admissions to hospitals, across eight countries, showed the United Kingdom figures to be similar to the median, yet computerisation is much less prevalent in the other countries. ${ }^{21}$

The control and transfer of information is another significant source of error. Much of prescribing involves copying the decision of another doctor, and if this is inaccurately communicated then prescribing errors ensue. This often happens at admission to, or discharge from, hospital; on transfer between general practices; and between doctors in a team. Recently the Hammersmith Hospital (and several others) has adopted a low technology solution to this. All patients on trial wards had a drug history taken by a 
pharmacist, then they were supplied all their drugs ready labelled and with a sufficient supply to take home once they were discharged. On average the pharmacists found 0.6 extra drugs per patient than the admitting doctor had, and the "allergy" box was filled 93\% of the time, compared with 50\% in the control wards. On discharge, simply providing a copy of the discharge prescription for the patient's community pharmacist can significantly reduce serious errors in medicine taking following the discharge of patients. ${ }^{22}$

A different form of control is that of access to, and monitoring of, riskier drugs and perhaps too "riskier" patients (those with little tolerance to the effects of an error). At present most drugs are treated in a similar manner except for those scheduled under the Misuse of Drugs Act. Yet when studying harm from medication error the drugs with low therapeutic indices are those most commonly involved, including, for example, digoxin, warfarin, and antiepileptics. However, changes are beginning to happen and, in the United Kingdom, access to potassium chloride injections has recently been more closely regulated. Some hospitals are targeting drugs such as methotrexate.

A common argument against computerised prescribing and decision support is that controlling actions by removing choice leads to dangerous de-skilling. We do not accept this. Firstly, if the skills were there in the first place, prescribing error would not be as common as it is. Secondly, many skills in our general life have been lost without detriment to the benefits of mechanisation and automation. Pharmacists no longer roll pills, doctors rarely write a prescription that is a formula for the pharmacist to make up and, in driving our cars, we no longer have the ability to double declutch (a necessary technique before synchromesh gears and automatic gearboxes). Fourthly, the prospects of using pharmacogenetic information will make current approaches untenable: no human brain will be able to integrate a patient's lifetime medical and drug records, the results of current and past laboratory results, and a full genome scan, all in the space of a typical 7 minute consultation. Finally, having decisions made by computers does not necessarily mean loosing skills, it just means that the skills must be taught and learned in different (and better) ways.

\section{CULTURE}

There are several ways in which the organisational culture contributes to the prevalence of prescribing errors. Firstly, the small amount of teaching in undergraduate courses, and the absence of teaching of doses to house officers, all send a message that these issues are not particularly important. Surely, if choosing the right drug, "for the right patient, at the right dose" matters, then it would be taught? This point is linked to the next point, that of implicit knowledge.

When professionals talk to each other about a patient's medicines, often they only make the name of the medicine explicit, leaving vital information about the dose, form, and frequency implicit. On a consultant ward round it is not unusual to hear something like "Put them on digoxin". There is no mention of checking that the dose will suit the body weight and renal function, nor a debate about starting with a loading dose, nor a check that the patient is not on interacting drugs, nor a suggestion about if and when the plasma concentration might (or should) be measured. When patients are reviewed on ward rounds it is very rare to see a consultant look at the drug chart to review the overall prescription, whether the drugs have been administered correctly, and whether the chart is a mass of crossing outs and conflicting information.

Much of a doctor's prescribing is personal to them and not debated in the open. Hence, it is hard for any reflective learning to occur. Pharmacists' correction of prescriptions is usually just discussed with the prescriber and the information does not come out into the team for them to discuss and learn from. Even in primary care general practitioners will rarely discuss details of their prescribing with colleagues.

Prescribing needs to be seen as an important act. To achieve this, senior staff, both medical and managerial, should overtly spend time on it. Prescribing mistakes need to be acknowledged, taken seriously, discussed openly, and action taken in a blame free culture. Pharmacists' collusion in the current low profile of prescribing mistakes, giving verbal feedback to the prescriber, or writing on sticky notes, must be stopped. Their interventions need to be written in the notes and form part of a team approach to improving quality.

There are also structural issues as to why prescribing error is so prevalent. In secondary care the power structure is created vertically by clinical area. Medicines, however, go across all clinical areas although they are not a major issue for many clinical directors. Medicines are everybody's and nobody's. Everybody uses them and has opinions about them, but there is rarely anybody with enough power or influence in either primary or secondary care to lead on them at a local level. The Audit Commission ${ }^{15}$ has recently tried to increase the role of pharmacists in medicine management in hospitals but we have yet to see whether its recommendations are widely adopted. In primary care the new primary care trusts will generally have a pharmacist advisor and although their initial agenda will probably be cost containment, improvements in specific areas of prescribing, such as asthma, are equally important. We need a pharmacist to review, and potentially prescribe, drugs in each practice if we are to make a significant reduction in preventable drug related hospital admissions. To be effective this activity would have to tackle the issue of non-adherence.

If we are to reduce the prevalence of prescribing error to any extent then we are going to have to change the culture, so medicines become seen as important. To do so we are going to have to treat medicines in special ways-by controlling access and use, and by ensuring competence in the prescribers. It will take time and resource but our future patients will reap the benefits.

\section{DISCUSSION}

Prescribing will change. Medicines offer so much help, can deliver so much harm, and are the most expensive element in health care, after staff costs. What is more, society as a whole both craves medicines, yet also fears them. Medicines are too important for the status quo to continue-we will have to work differently.

The role of the doctor will change in a way that parallels changes in the theatre a century ago. The doctor will move from actor-manager to director (ideally, co-directing with the patient). The doctor, instead of deciding what should be done and delivering it her-/himself, will define the ethos and the ends of treatment, and use others to deliver them.

The diagnosis and the direction of treatment will be agreed between the doctor and patient, and then handed over to others, generally pharmacists, to choose the best drug and dose in collaboration with the patient, and to monitor and amend the drug and dose in response to its effects on the patient. The routine, technical/pharmacological tasks in prescribing will, in the way of all developing technologies, be taken over by specialists such as the pharmacist, and by improved technology. The doctor will still require skills for the solution of the more difficult problems in prescribing, particularly in hospitals, but not for routine prescribing.

Pharmacists and nurses who prescribe for patients will work more in therapeutic partnership with the patientagreeing the end point of treatment and using their knowledge of medicines to deliver it. Prescribing for acute 


\section{Key messages}

- Prescribing error is unacceptably frequent.

- Prescribers from all professions should be trained and proven competent.

- Technology should be used to guide the prescriber to the correct prescription for that patient.

- The importance of prescribing needs to be raised and champions of medicine use are needed.

- The role of the doctor will increasingly become to direct therapy delivered and monitored by others.

conditions will become more the work of nurses and community pharmacists.

Practices and hospitals will have a "director of prescribing", who will monitor and develop prescribing and prescribers. They will have the power to change staff and systems. Until such fundamental differences in structure and culture are achieved, the benefits of devolving prescribing will be limited.

\section{Authors' affiliations}

N Barber, The School of Pharmacy, 29-39 Brunswick Square, London WCIN IAX, UK

M Rawlins, Wolfson Unit of Clinical Pharmacology, University of Newcastle, Newcastle upon Tyne NE2 4HH, UK; National Institute for Clinical Excellence, MidCity Place, 71 High Holborn, London WCIV 6NA, UK

B Dean Franklin, Academic Pharmacy Unit, Hammersmith Hospitals NHS Trust and The School of Pharmacy, Hammersmith Hospital, London W12 OHS, UK

\section{REFERENCES}

1 Kohn T, Linda T, Corrigan M, et al. To err is human: building a safer health system. Washington DC: National Academy Press, 2000.
2 Hepler CD, Segal R. Preventing medication errors and improving drug therapy outcomes: a management systems approach. Boca Raton, Florida: CRC Press LLC, 2003.

3 Dean B, Schachter M, Vincent C, et al. Prescribing errors in hospital inpatients: their incidence and clinical significance. Qual Saf Health Care 2002;11:340-4.

4 Sanders J, Esmail A. The frequency and nature of medical error in primary care: understanding the diversity across studies. Fam Pract 2003;20:231-6.

5 Omori DM, Potyk RP, Kroenke K. The adverse effects of hospitalization on drug regimens. Arch Intern Med 1991;151:1562-4.

6 Meichenbaum D, Turk DC. Facilitating treatment adherence: a practitioner's handbook. New York: Plenum Press, 1987.

7 Sackett DL, Snow JC. The magnitude of compliance and non-compliance. In: Haynes RB, Taylor WD, Sackett DL, eds. Compliance in health care. Baltimore, London: The John Hopkins University Press, 1979:11-22.

8 The Association of the British Pharmaceutical Industry. Facts \& statistics from the Pharmaceutical Industry: 4-Medicines and the NHS, http:// www.abpi.org.uk/statistics/section.asp? sect = 4, 2003

9 Reason J. Human error. Cambridge: Cambridge University Press, 1990.

10 Rawlins MD. Making tomorrows doctors better prescribers. Br J Clin Pharmacol 2003:55:495.

11 Maxwell S, Walley T. Teaching safe and effective prescribing in UK medical schools a core curriculum for tomorrows doctors. Br J Clin Pharmacol 2003;55:496-503

12 Maxwell S, Walley T, Ferner RE. Using drugs safely: undergraduates must be proficient in basic prescribing. BMJ 2002;324:930-1.

13 Ellis A. Prescribing rights: are medical students properly prepared for them? BMJ 2002;324:1591.

14 Callum KG, Carr NJ, Gray AJG, et al. The 2002 report of the national confidential enquiry into perioperative deaths. London: National Confidential Enquiry into Perioperative Deaths, 2002.

15 The Audit Commission. A spoonful of sugar: medicines management in NHS hospitals. London: The Audit Commission, 2002.

16 Dean BS, Schachter M, Vincent C, et al. Causes of prescribing errors in hospital inpatients: a prospective study. Lancet 2002;359:1373-8.

17 BMJ, Royal Pharmaceutical Society of Great Britain. British national formulary. Wallingford and London: Pharmaceutical Press, BMJ Books.

18 Barber N, Batty R, Ridout D. Predicting the rate of physician-accepted interventions by hospital pharmacists in the United Kingdom. Am J Health Syst Pharm 1997; 54:397-405.

19 Bates DW, Teich JM, Lee J, et al. The impact of computerized physician order entry on medication error prevention. J Am Med Inform Assoc 1999;6:313-21.

20 Sagripanti M, Dean BS, Barber N. An evaluation of the process-related medication risks for elective surgery patients from pre-operative assessment to discharge. International Journal of Pharmacy Practice 2002;10:161-70.

21 Winterstein A. Preventable drug-related hospital admissions and morbidity in hospitalized patients: a meta-analysis of prevalence reports. Ann Pharmacother 2002;36:1238.

22 Duggan C, Feldman R, Hough J, et al. Reducing adverse prescribing discrepancies following hospital discharge. International Journal of Pharmacy Practice 1998:6:77-82. 